OPEN ACCESS

$$
\begin{array}{r}
\text { Edited by: } \\
\text { Huiyu Zhou, } \\
\text { University of Leicester, } \\
\text { United Kingdom } \\
\text { Reviewed by: } \\
\text { Guodong Zeng, } \\
\text { University of Bern, Switzerland } \\
\text { Shuyang Chen, } \\
\text { ABB, United States } \\
\text { *Correspondence: } \\
\text { Anmin Zhu } \\
\text { azhu@szu.edu.cn }
\end{array}
$$

Received: 20 October 2021 Accepted: 30 December 2021

Published: 11 February 2022

Citation:

Yi X, Zhu A and Yang SX (2022) MPPTM: A Bio-Inspired Approach for Online Path Planning and High-Accuracy Tracking of UAVs.

Front. Neurorobot. 15:798428.

doi: 10.3389/fnbot.2021.798428

\section{MPPTM: A Bio-Inspired Approach for Online Path Planning and High-Accuracy Tracking of UAVs}

\author{
Xin $\mathrm{Yi}^{1}$, Anmin Zhu ${ }^{1 *}$ and S. X. Yang ${ }^{2}$ \\ ${ }^{1}$ Research Institute of Intelligence Technology and System Integration, College of Computer Science and Software \\ Engineering, Shenzhen University, Shenzhen, China, ${ }^{2}$ Advanced Robotics and Intelligent Systems (ARIS) Laboratory, School \\ of Engineering, University of Guelph, Guelph, ON, Canada
}

The path planning and tracking problem of the multi-robot system (MRS) has always been a research hotspot and applied in various fields. In this article, a novel multi-robot path planning and tracking model (MPPTM) is proposed, which can carry out online path planning and tracking problem for multiple mobile robots. It considers many issues during this process, such as collision avoidance, and robot failure. The proposed approach consists of three parts: a neural dynamic path planner, a hyperbolic tangent path optimizer, and an error-driven path tracker. Assisted by Ultra-wideband positioning system, the proposed MPPTM is a low-cost solution for online path planning and high-accurate tracking of MRS in practical environments. In the proposed MPPTM, the proposed path planner has good time performance, and the proposed path optimizer improves tracking accuracy. The effectiveness, feasibility, and better performance of the proposed model are demonstrated by real experiments and comparative simulations.

Keywords: multi-robot system, path planning, neural dynamics, path tracking, neural network

\section{INTRODUCTION}

As the development of disciplines and technologies, robotics always involves numerous disciplines. It covers many aspects from control, mechanics, electronics to communication, computer science, materials, and so forth. Robotics has also developed from a simple single robot system (SRS) to a complex multi-robot system (MRS). For dealing with complex problems, MRS has more advantages than SRS. Large numbers of researches state that the cooperation of MRS has been applied to more practical fields, such as services (Morita et al., 2018; Krizmancic et al., 2020), therapy (Ali et al., 2019; Mehmood et al., 2019), rescue (Queralta et al., 2020), training (Xu and Tang, 2021), and so on.

Path planning and tracking problem of MRS has always been a research hotspot and applied in various fields, including delivery (Chen et al., 2021), monitoring (Silic and Mohseni, 2019; Koutras et al., 2020), task assignment (Chen and Zhu, 2019; Wang et al., 2020b), target tracking (Zhou et al., 2018), and so on. In order to solve this problem, many kinds of research can be divided into three aspects: task-level, control-level, and task-control-level (Zeng et al., 2015; Rubí et al., 2019). The task-level research focuses on finding the optimal solution to the problem without considering the hardware conditions, which is top-down. It includes path planning of MRS and task assignment of MRS. The control-level research needs to consider the hardware of MRS and the realization of the solution, which is down-top. Tracking the target or path with high accuracy is one of these 
kinds of research. The task-control-level research combines tasklevel and control-level, and the path planning and tracking problem is one of this kind of research.

In the task-level research of MRS, path planning and task assignment are the two mainstreams. Compared with task assignments, path planning is more focused on the time-space continuity and process. For example, these studies (Yi et al., 2017; Dai et al., 2019; Ali et al., 2020; Dong et al., 2020; Han and Yu, 2020; Wang et al., 2020a) only consider task-level without considering control-level details. Combining with deep learning, (Wang et al., 2020a) proposed a neural $\mathrm{RRT}^{\star}$ for path planning of MRS, but it needs a lot of processed data for training before path planning (Wang et al., 2020a). Han et al. used database heuristics for fast near-optimal path planning of MRS, it can carry out efficient path planning, but its applicable scene is only based on grid environment (Han and Yu, 2020). Yi et al. proposed a bio-inspired approach to plan the path of robots in 3-D environments. Also, it can make real-time path planning but can not avoid obstacles (Yi et al., 2017). Dong et al. proposed a path planning method of UAVs in the 3-D environment for the inspection of transmission lines. But it is only made available for a single target, not for multiple targets (Dong et al., 2020).

Path tracking for MRS is solved by control-level algorithms. These algorithms tend to reduce error during the tracking process while considering the difference of robot hardware in MRS. Ma proposed cooperative tracking of MRS with circular formation, but it can not track multi-target (Ma, 2020). Yu et al. proposed a formation tracking method based on an adaptive neural network, but it just makes MRS formate to track a single target (Yu et al., 2018). Zhou et al. presented a resilient tracking method for MRS. It is suitable for patrol and monitoring in the area but can not track the immovable target (Zhou et al., 2018).

Both task-level and control-level studies are very limited in practical application. Therefore, some studies focusing on both task-level and control-level have occurred.

Park et al. proposed a distributed approach combing alternating direction method of multipliers (ADMM) to nonmyopic path planning for multi-target tracking, but it can not avoid obstacles in the environment (Park et al., 2019). Yordanova et al. proposed a path planning and tracking method for the area coverage of autonomous underwater vehicles, but in essence, the method is still only for the 2-D environment without obstacles (Yordanova and Gips, 2020). Penin et al. proposed a visionbased path planning and target tracking method for UAVs (Penin et al., 2018). It can deal with collision avoidance and obstacle avoidance, but its accuracy of vision-based positioning is still questionable for indoor environments.

Compared with these studies (Penin et al., 2018; Park et al., 2019; Yordanova and Gips, 2020; Yu et al., 2020), there are few studies for online path planning and tracking of MRS. During online path planning and tracking of MRS, the proposed model needs to plan the trajectories easy to track in real-time, which deals with dynamic environments and accidents, such as robot fault, moving obstacles, and so on. Online path planning and tracking need to solve the following three problems. (1) How can path planning meet the requirements of real-time; (2) How can the planned paths be transformed into trajectories easy to track; and (3) How to efficiently organize related processes?

In this study, a novel model named MPPTM (multirobot path planning and tracking model) is proposed for online path planning and high-accuracy tracking of MRS. It does not depend on the sensors of the individual robot in MRS by using an Ultra-wideband (UWB) positioning system. Therefore, the proposed approach is a low-cost solution for warehouse or factory environments. The proposed model has the following innovations.

1. The proposed model uses superscalar pipelining mode to organize these processes more efficiently. Therefore, the process of path tracking does not need to wait until the end of path planning.

2. Compared with traditional path planners, the proposed neural dynamic path planner has better time performance.

3. In our proposed model, the hyperbolic tangent path optimizer bridges the planned paths and the trajectories easy to track, and it reduces the tracking error of MRS.

The remainder of the article is organized as follows. In section 2 , the components and framework of the proposed model are introduced in detail. The experiments for MRS in a 3-D environment are present in section 3. Some further discussions about the comparative studies are given in section 4 . Finally, the conclusion and future study are presented in section 5 .

\section{PROPOSED APPROACH}

The proposed MPPTM is described in detail in this section. It is applicable for not only $2-\mathrm{D}$ but also $3-\mathrm{D}$ environments. It combines the task-level and control-level, which can deal with the path planning of MRS, and cope with the path tracking of MRS.

The proposed MPPTM mainly integrates three parts, including the path planner, the path tracker, and the path optimizer. The path planner is responsible for dealing with the online path planning of MRS at the task-level. During the path planning of MRS, obstacles avoidance, collision avoidance, and other robot accidents are considered. The path tracker is used to cope with the path tracking of MRS. During the path tracking of MRS, reducing tracking error and compatibility with different hardware are considered. The path optimizer is used to bridge the gap between the path planner and the path tracker, which makes the planned path to be easier tracked. It can process the online path planning and path tracking for MRS in complex environments.

The framework of MPPTM with environments is shown in Figure 1. The process of path planning and tracking, the cooperation mechanism between the path planner and the path tracker are given in the framework of MPPTM. In Figure 1, $k$ and $t$ are the iterations of the planner and the iteration of the tracker, respectively. For the path planner, $I(k)$ represents the environmental information, and $P_{c}(k)$ represents the current posture of the MRS. Both are used to generate the desired posture $P_{d}(k)$ through $Q(k)$. Through the path optimizer, the desired posture $P_{d}(k)$ is transferred into the desired trajectory $T_{d}(t)$ for 


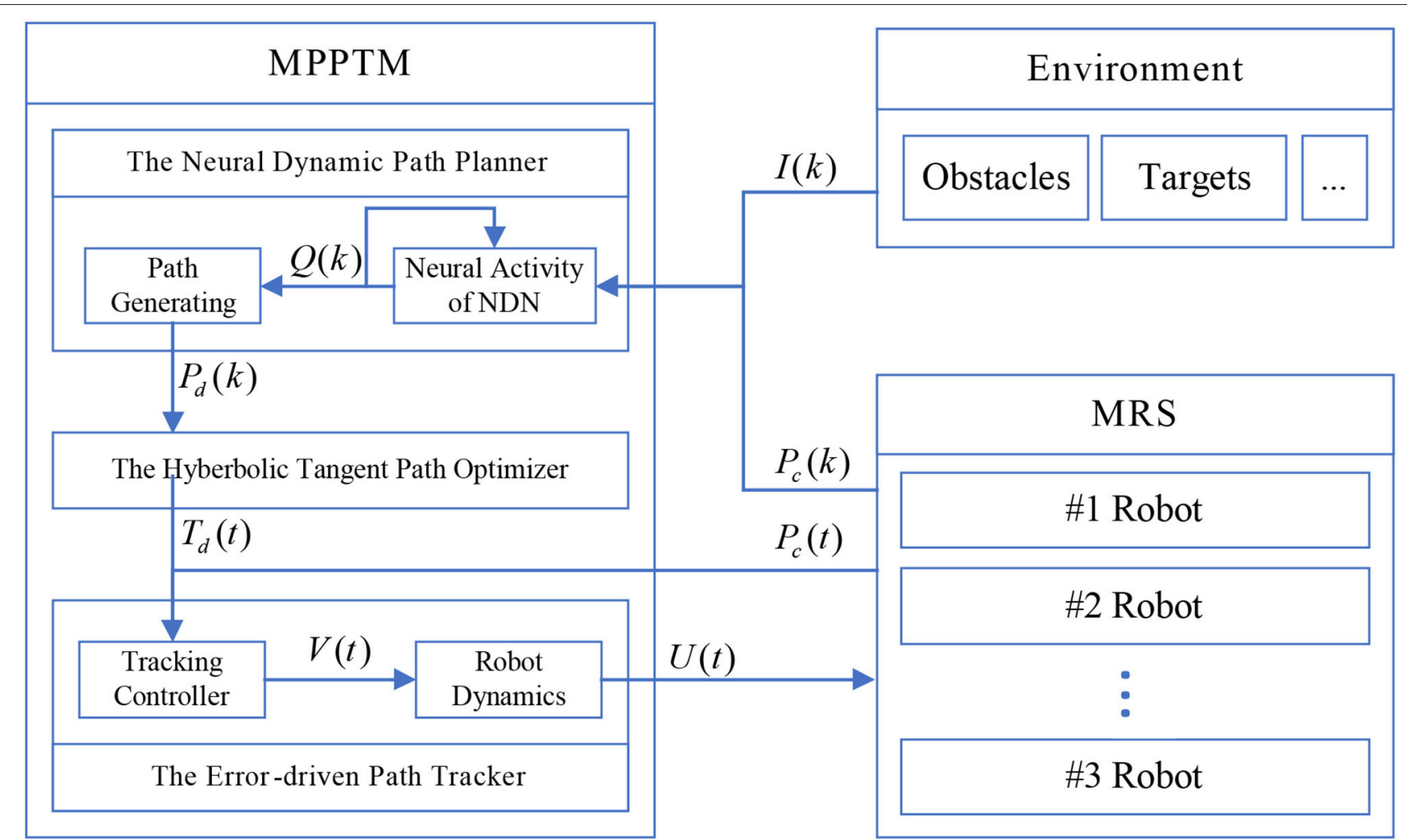

FIGURE 1 | Overall schematic diagram of multi-robot path planning and tracking model (MPPTM) with environments.

the path tracker to track. In the path tracker, the desired posture $T_{d}(k)$ and current posture $P_{c}(t)$ of MRS are used to generate the desired velocity $V(t)$ by path tracker for MRS. Additionally, then it is converted into the desired motor speed $U(t)$ by robot dynamics. In the MRS, each robot tries to achieve the desired motor speed $U(t)$ to move.

Usually, there are two different modes to deal with the path planning and tracking of MRS, which are superscalar pipelining mode and traditional mode.

In traditional mode, path tracking must wait until all path planning and optimization are completed. This mechanism handles these processes serially, which can save computing resources. However, it is only suitable for path planning and tracking of MRS in static environments.

Due to the time performance of the proposed path planner, the proposed MPPTM applies superscalar pipelining mode to deal with these processes, such as path planning, path optimizing, and path tracking, as shown in Figure 2.

In superscalar pipelining mode, part of the planned path is used to be optimized by the path optimizer, and tracked by the path tracker. Path tracking does not have to wait for all path planning and optimization to be completed. This mechanism parallels these processes to some extent, which can be suitable for online path planning and tracking of MRS in dynamic environments.
The proposed MPPTM is introduced in detail by three following parts: (A) The neural dynamic path planner; (B) The error-driven path tracker; and (C) The hyperbolic tangent path optimizer.

\subsection{The Improved Neural Dynamic Path Planner}

As an important part of MPPTM, the neural dynamic path planner is used to plan paths for MRS. It is based on neural dynamics and has the advantages and characteristics of the biological neural system. The neural dynamic path planner for 3-D environments is introduced in the section, and the proposed path planner for 2-D environments is similar to this. The neural dynamic path planner includes three following parts.

\subsubsection{The Neural Dynamic Network (NDN)}

In the neural dynamic path planner, the NDN is used to real-time describe the environment where MRS is located. In NDN, the distance between any two adjacent neurons is equal, its distance is 1 , and any two adjacent neurons are also connected to each other. The structure of NDN describing the 3-D environment is as shown in Figure 3.

The activity of neuron $q_{i j k}$ is in the $i$ th row, the $j$ th column, and the $k$ th page of the NDN, which describes the environment that it maps. The activities of NDN $Q$ 

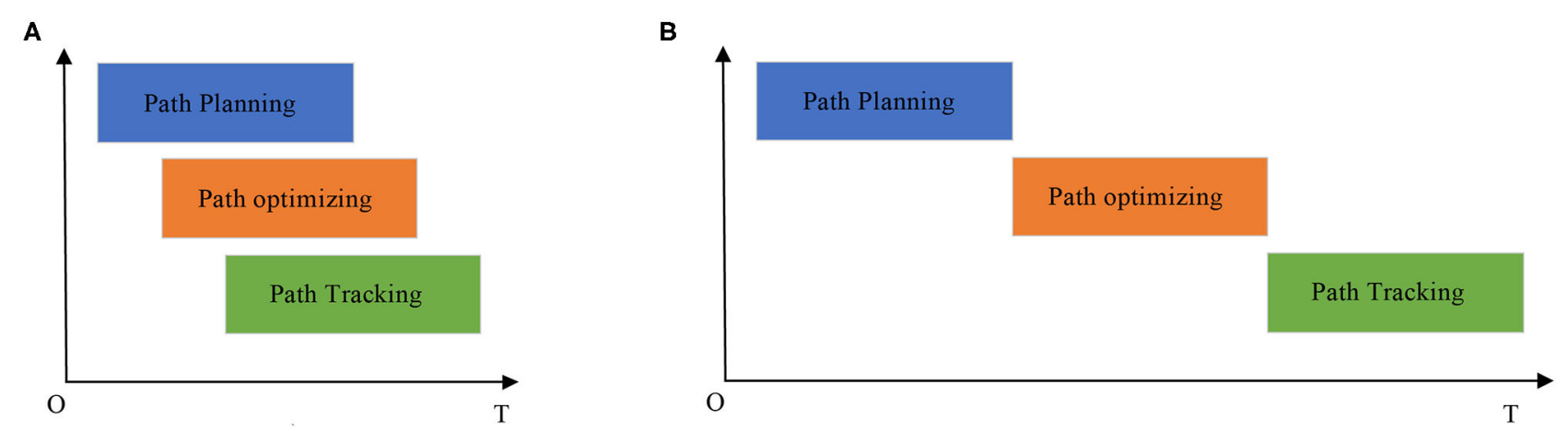

FIGURE 2 | Two different operation modes in MPPTM. (A) The superscalar pipelining mode. (B) The traditional mode.

describing 3-D environments is a 3-D matrix defined by Equation (1).

$$
Q=\left[\begin{array}{c}
{\left[\begin{array}{ccc}
q_{111} & \cdots & q_{1 n 1} \\
\vdots & \ddots & \vdots \\
q_{m 11} & \cdots & q_{m n 1}
\end{array}\right]} \\
\vdots \\
{\left[\begin{array}{ccc}
q_{11 h} & \cdots & q_{1 n h} \\
\vdots & \ddots & \vdots \\
q_{m 1 h} & \cdots & q_{m n h}
\end{array}\right]}
\end{array}\right]_{m \times n \times h}
$$

In Figure 3, $r$ is the radius of the range that neurons can affect, $x$ is horizontal offset, $y$ is vertical offset, $z$ is longitudinal offset, and $x, y, z$ are integers. The radius of the search sphere $r$ directly affects the computation performance and the accuracy of path planning. If radius $r$ is set too large, the planned path will pass through the obstacles in the environment. In NDN, the activity of each neuron $q$ represents the environment where it maps. Therefore, the activities of NDN $Q$ describe the whole environment.

\subsubsection{The Improved Neural Activity Algorithm}

This algorithm states the activity of neural signals in NDN. Through multiple iterations of neural activity, the activities of NDN $Q$ tend to be stable, and all kinds of signals have been fully spread in NDN. The neural activity of NDN is the core part of the proposed path planner, and it is also the most timeconsuming process. Therefore, we proposed an improved neural activity algorithm of NDN, and it is defined by Equation (2).

$$
\begin{aligned}
\frac{d Q}{d t}= & -K Q+(D-Q)\left([I]^{+}+[F(x, y, z)]^{+}\right) \\
& -(J+Q)\left([I]^{-}+[F(x, y, z)]^{-}\right)
\end{aligned}
$$

Three parameters, $K, D, J$, are decay rate, upper bound, and lower bound, respectively, in the dynamic equation of neural activity. Meanwhile, two operators, $[a]^{+}$and $[b]^{-}$, obtain, respectively, $\max \{a, 0\}$ and $\max \{-a, 0\}$. The function $F(x, y, z)$ is the weighted sum of the shifting matrix $Q$ with these offsets $x, y, z$, and it is defined by Equation (3).

$$
F(x, y, z)=\sum_{x y z} w_{x y z} \operatorname{shift}(Q, x, y, z) \sqrt{x^{2}+y^{2}+z^{2}} \leq r
$$

where $\operatorname{shift}(Q, x, y, z)$ shifts the elements of matrix $Q$ with the $x$ rows, the $y$ columns, and the $z$ pages, but it satisfies the condition $\sqrt{x^{2}+y^{2}+z^{2}} \leq r$. This weight $w_{x y x}$ is defined by Equation (4).

$$
w_{x y z}=\frac{u}{\sqrt{x^{2}+y^{2}+z^{2}}} \quad \sqrt{x^{2}+y^{2}+z^{2}} \leq r
$$

where $w_{x y z}$ is the connection weight when the horizontal offset is $x$, the vertical offset is $y$, and longitudinal offset is $z$ in $\mathrm{NDN}, u$ is the positive parameter and represents the intensity of the connection.

In Equation (2), environmental information $I$ is a $3-D$ matrix with the same size as $Q$, its element is defined as Equation (5). Ex and $I n$ are positive parameters, which represent the intensity of excitatory nerve signal and inhibitory nerve signal, respectively, in NDN.

$$
i_{i j k}=\left\{\begin{array}{cc}
E x & \text { The neuron maps target } \\
- \text { In } & \text { The neuron maps robot } \\
0 & \text { Others }
\end{array}\right.
$$

\subsubsection{The Path Generation for 3-D Environments}

After the multiple iterations of neural activity, the activities of NDN $Q$ are used to generate the next position during the path planning of MRS. The next position of the $i_{t h}$ robot $P^{i}(k+1)$ is defined as Equation (6).

$$
P^{i}(k+1) \Leftarrow q_{a b c}=\max \left\{q_{e f g} \mid 0<\left\|q_{e f g}-q_{i j k}\right\|<r\right\}
$$

Assume that the current position of the $i_{t h}$ robot is mapped by the neuron $q_{i j k}, q_{\text {efg }}$ is the set of neurons in the affected range of the neuron $q_{i j k}$. In the set $q_{\text {efg }}$, the maximum activity of the neuron $q_{a b c}$ is selected as the next position $P^{i}(k+1)$ of the $i_{t h}$ robot during path planning.

\subsection{The Error-Driven Path Tracker}

The proposed path tracker uses error driven method to track the path planned by the proposed path planner. The error-driven path tracker is capable of being compatible with a variety of controllers and the hardware of MRS. In order to better test the performance of the proposed MPPTM in 3-D environments, a 


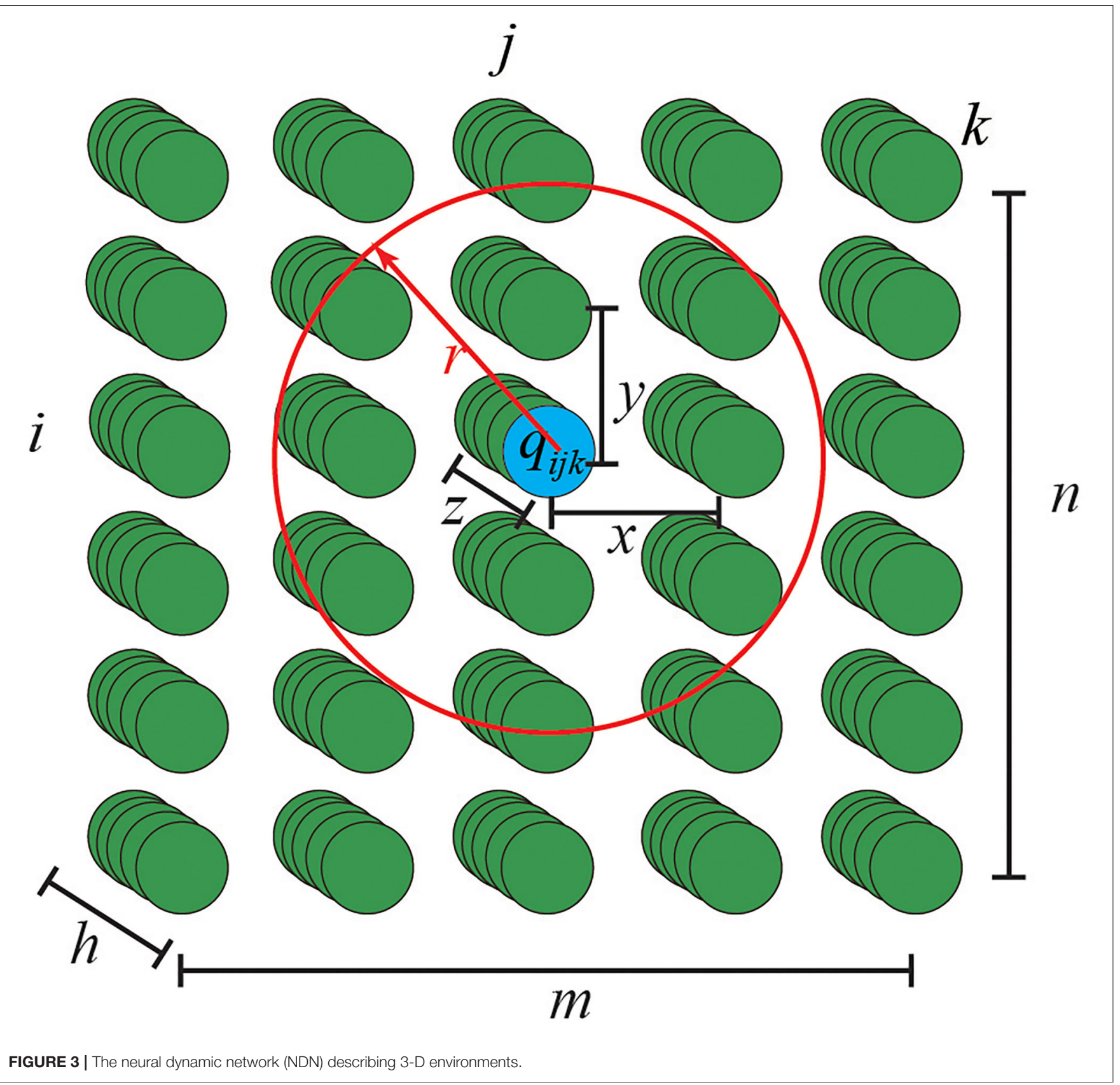

path tracker with PID controller for quadrotor is given briefly in the article, which is used in the following experiments.

Quadrotor, a helicopter with four rotors, is a small unmanned aerial vehicle (UAV). Additionally, it is used as an individual MRS in 3-D environments. Two control loops with PID control are used in the path tracker of MRS for path tracking in 3$\mathrm{D}$ environments. The system architecture of the proposed path tracker for 3-D tracking with PID control is shown in Figure 4.

\subsection{The Hyperbolic Tangent Path Optimizer}

The path optimizer bridges the gap between the path planner with the path tracker, it translates the task-level paths planned by the path planner into the control-level paths for the path tracker. During this process, the task-level paths $P^{i}(k)$ with low frequency should be transformed into the control-level trajectory $T^{i}(t)$ with high frequency, which can make the planned paths easy to track for MRS. The planned paths are given by the path planner are defined as Equation (7), where $\operatorname{Paths}^{i}(\gamma)$ is the continuous planned path of the $i_{\text {th }}$ robot in MRS, $k$ is the iteration of the path planner, and $R$ is the number of robots in MRS. 


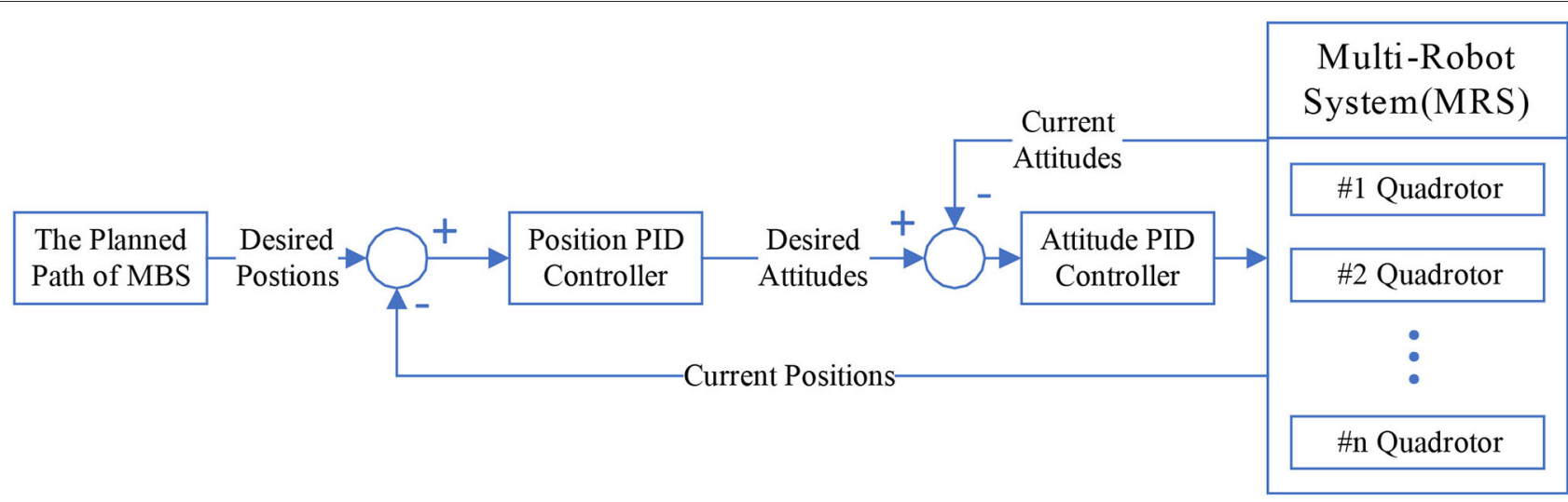

FIGURE 4 | The proposed path tracker for quadrotors in 3-D environments.

The control-level trajectory $T_{o s}^{i}(t)$ given by the path optimizer with original sampling from $P^{i}(k)$ can be obtain by Equation (8), where the operator $\lfloor a\rfloor^{b}$ obtains the largest element in set $b$ and less than or equal to $a$, and $w$ is the sampling frequency.

$$
\left\{\begin{array}{c}
a=\lfloor t / w\rfloor^{k} \\
T_{o s}^{i}(t)=\left[P^{i}(a+1)-P^{i}(a)\right]^{\prime} \times \frac{t \% w}{w} \quad i=1,2 \ldots R
\end{array}\right.
$$

In order to improve the tracking performance of MRS, an improved sampling method is proposed in the article. $P^{i}(\tau)$ is the set of elements in set $P^{i}(k)$ whose elements are not differentiable on the paths of MRS, which can describe as Equation (9).

$$
\left.\begin{array}{l}
P^{i}(\tau) \in\left\{P^{i}(k) \mid \lim _{\Delta \delta \rightarrow 0^{+}} \dot{P}^{i}(k+\Delta \delta) \neq \lim _{\Delta \delta \rightarrow 0^{-}} \dot{P}^{i}(k+\Delta \delta)\right. \\
k \in 1,2 \ldots, n
\end{array}\right\}
$$

The trajectory $T_{h t s}^{i}(t)$ given by the path optimizer with hyperbolic tangent sampling from $P^{i}(\tau)$ can be obtain by Equation (10), where the operator $\lceil a\rceil^{b}$ obtains the smallest element in set $b$ and more than or equal to $a, w$ is the sampling frequency.

$$
\left\{\begin{array}{c}
a=\lceil t / w]^{\tau} \\
b=[t / w]^{\tau} \\
T_{h t s}^{i}(t)=\left[P^{i}(a)-P^{i}(b)\right] \times f\left(\frac{t-w \times b}{w \times(a-b)}\right) \quad i=1,2 \ldots R
\end{array}\right.
$$

where $f(x)$ is the hyperbolic tangent function defined as Equation (11). Optimal trajectory with hyperbolic tangent sampling $T_{h t s}^{i}$ is generated by optimizing the path $\operatorname{Paths}^{i}(\gamma)$ according to the referenced velocity.

$$
f(x)=0.5 \times\left(\frac{e^{2 x+1}-1}{e^{2 x+1}+1}+1\right)
$$

Compared with the path planner with original sampling, the one with hyperbolic tangent sampling can give the trajectory which is easier to track for MRS, which is demonstrated in the following experiment.

\section{EXPERIMENTS}

For demonstrating the feasibility of the proposed MPPTM, online path planning and tracking experiments for multi-UAV are designed in this section. However, it should be noted that the proposed MPPTM is suitable for online path planning and tracking of MRS not only in 2-D environments but also in 3-D environments.

\subsection{Experimental Preparations}

In order to accurately locate UAV groups in 3-D environments, the UWB positioning system is used, as shown in Figure 5. At least four UWB locator nodes are used to locate UAV groups in 3-D environments, and the distance between two adjacent nodes is $15 \mathrm{~m}$. A micro quadrotor produced by Zeronetech is used as an individual of the UAV group in this experiment, and it has a built-in UWB tag and wireless communication module.

The laptop with R5800u CPU and 32GB ROM is responsible for collecting the location data of UAVs via the UWB positioning system, recoding the flight data of UAVs, and controlling the fight of UAVs via WiFi communication. The system is implemented by Matlab and $\mathrm{C}++$, the proposed path planner is coded by Matlab, the proposed path optimizer and the proposed path tracker are coded by $\mathrm{C}++$. The obstacles in 3-D environments are realized by marking the environmental information $I(k)$ in the proposed path planner.

\subsection{Online Path Planning and Tracking for 3-D Environments}

The size of the outdoor environment used for testing is $10 \mathrm{~m} \times 10 \mathrm{~m} \times 10 \mathrm{~m}$, which is mapped by the NDN with size $50 \times 50 \times 50$ in the neural dynamic path planner. In the proposed path planner, we set $K$ to $50, D$ to $5, J$ to 3, $u$ to $0.3, r$ to 2, Ex to 50, and $I n$ to 5 . In the proposed path optimizer, hyperbolic tangent sampling is used at the condition $w=100$. The initial positions of targets and quadrotors are randomly located. The initial sizes 


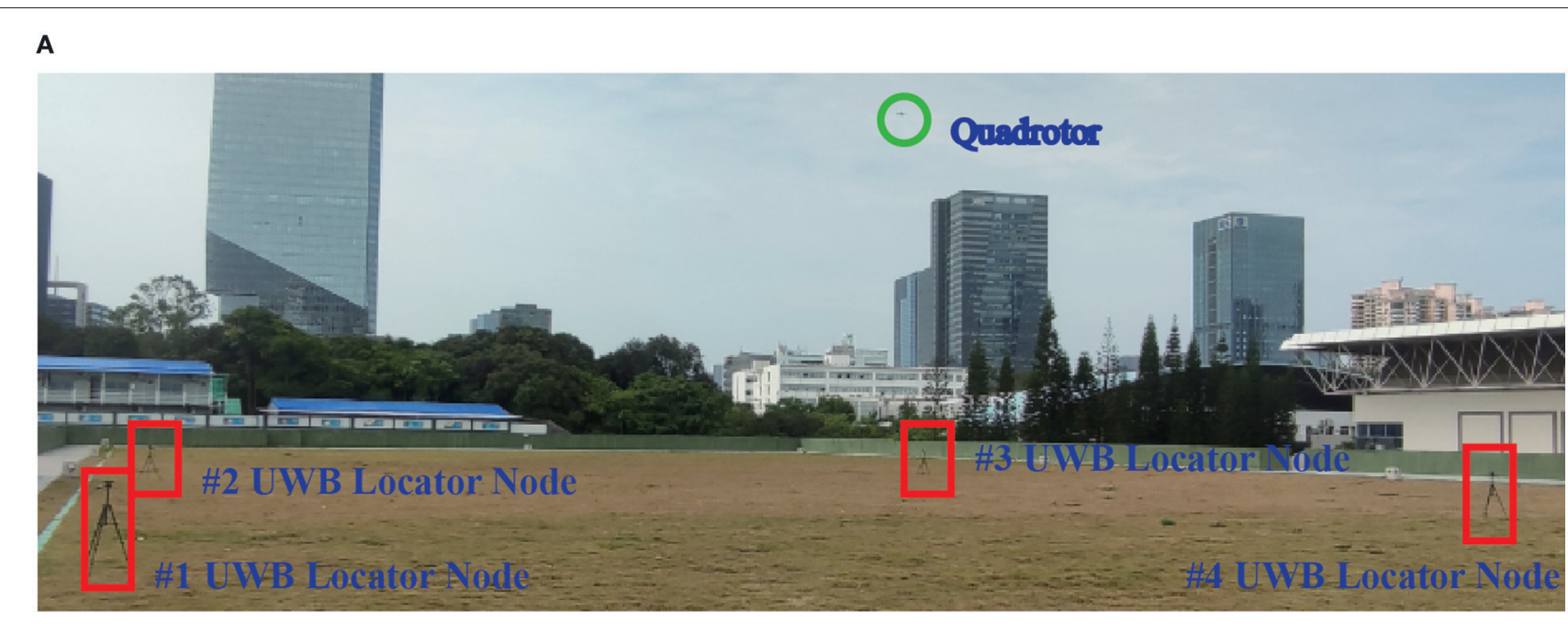

B

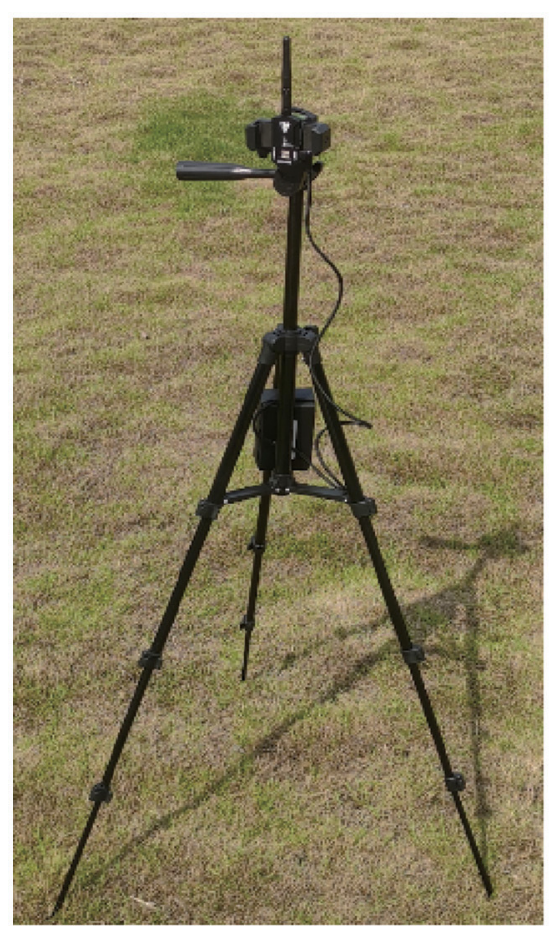

C

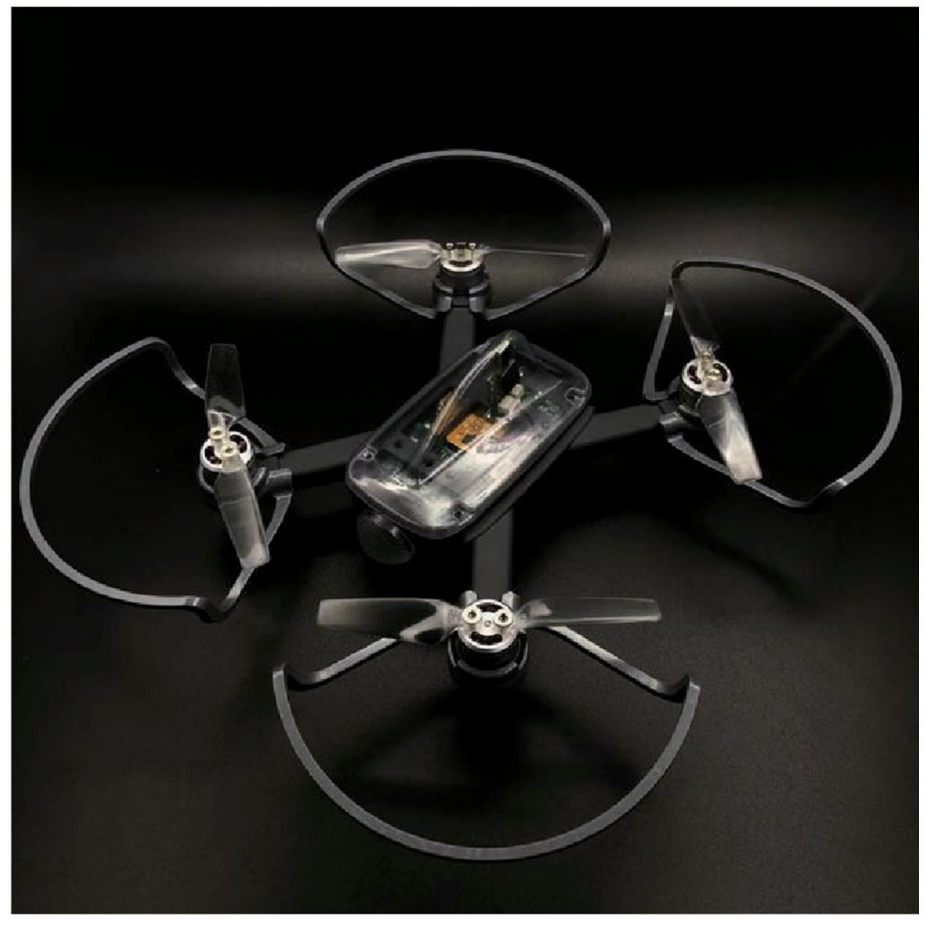

FIGURE 5 | The experimental preparations for online path planning and tracking. (A) The experimental site for online path planning and tracking of multi-UAVs. (B) The UWB locator node. (C) A micro quadrotor with UWB tag.

and initial positions of obstacles are randomly generated to mark the environmental information $I(k)$.

The snapshot of path planning and tracking at $30 \mathrm{~s}$ is shown in Figure 6, and the 1th quadrotor has finished capturing the target. Figure 7 shows the snapshot of path planning and tracking at $65 \mathrm{~s}$, where all quadrotors have captured targets.

The velocities of quadrotors in $X, Y, Z$ directions are recoded and shown in Figure 8. The experimental result shows that all quadrotors can avoid collision and obstacles, and capture targets. The experimental result demonstrates that MPPTM is capable of online path planning and tracking of MRS in 3-D complex environments.

\subsection{Online Path Planning and Tracking With Robot Fault}

This experiment uses the same parameters and the same environment from the experiment in section 3.2. But in the online path planning and tracking, there are two quadrotors that have a certain probability 0.05 of failure during the process. Additionally, MPPTM needs to allocate 

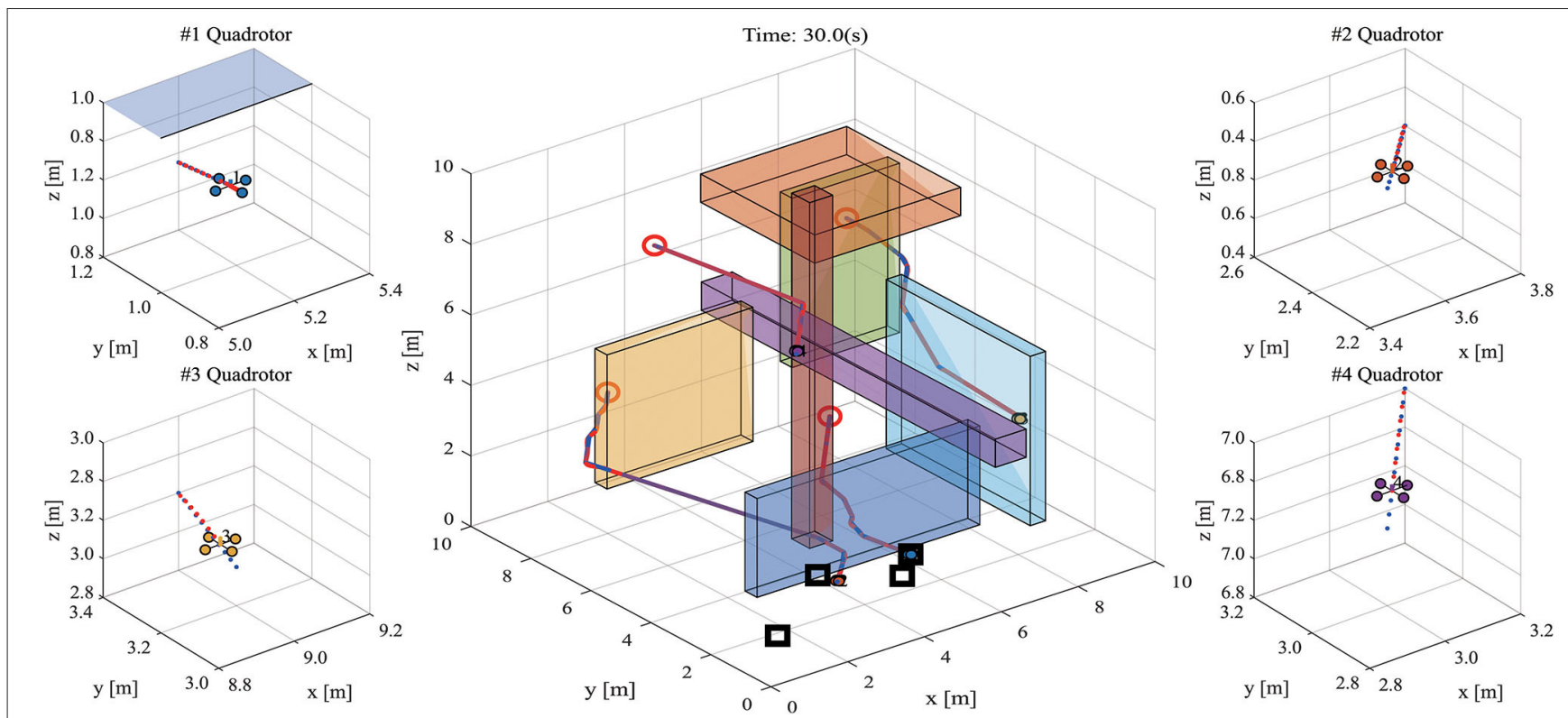

FIGURE 6 | The snapshot of path planning and tracking for UAVs in 3-D environments at $30 \mathrm{~s}$. (Red circles and black squares present the initial positions of robots and targets respectively on the main diagram. Four sub diagrams zoom in the main diagram and show the current flight states of quadrotors. The red dot line presents the real path and the blue dot line presents the referenced path).
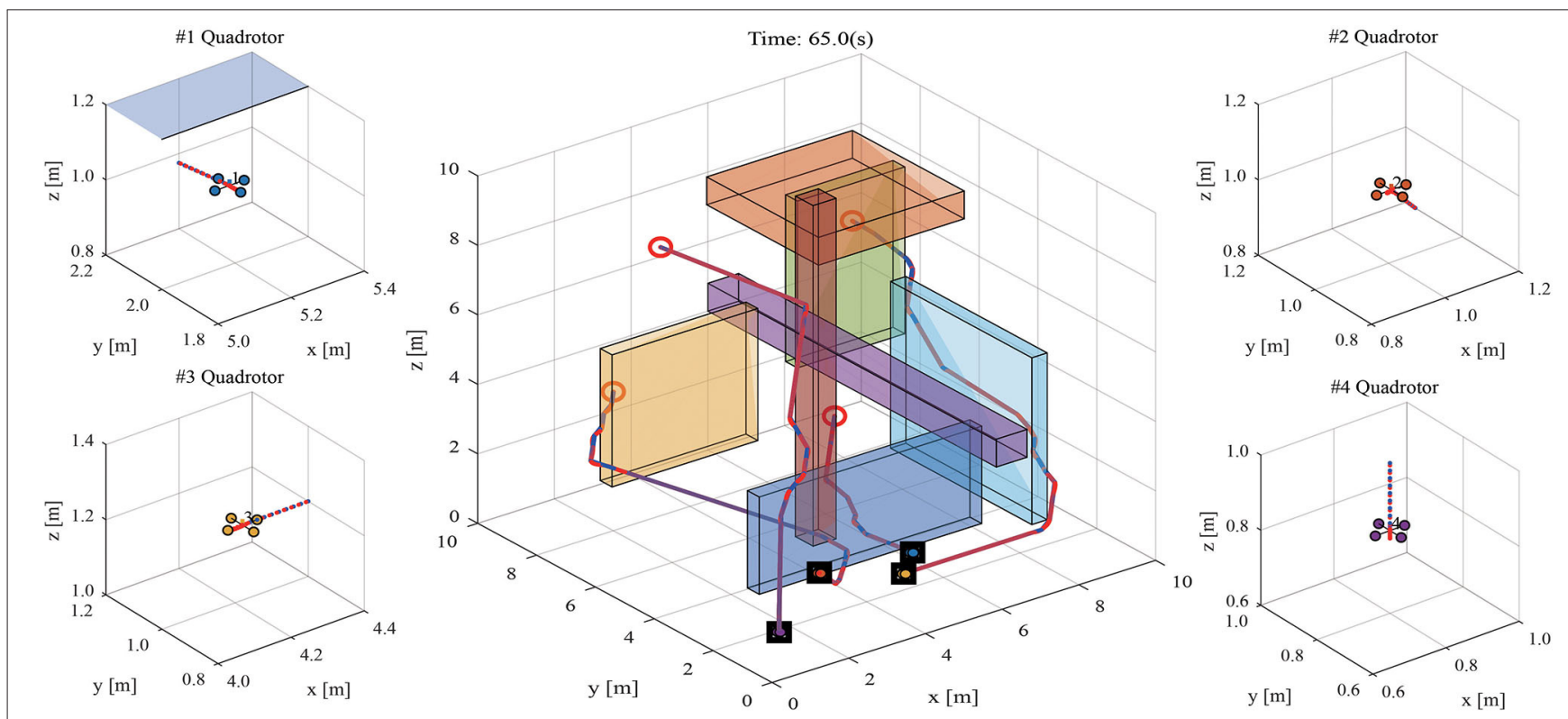

FIGURE 7 | The snapshot of path planning and tracking for UAVs in 3-D environments at $65 \mathrm{~s}$. (The meaning of markers is the same as that in Figure 6).

these two targets to the other two quadrotors during the process.

As shown in Figure 9, after two quadrotors break down, the other quadrotors have also successfully completed four targets capturing. Therefore, the Experimental result indicates that MPPTM can deal with robot fault during online path planning and tracking, and it can reassign targets timely after robot fault.

\subsection{Online Path Planning and Tracking With Dynamic Obstacles}

This experiment is conducted in the 3 -D workspace $8 \mathrm{~m} \times 2.5 \mathrm{~m} \times$ $2.5 \mathrm{~m}$, and the experimental environment is mapped by the NDN with size $40 \times 10 \times 10$. The experimental parameters are the same as those of the experiment in section 3.2. Obstacles in this experiment move at different times $(8 s, 14 s, 28 s)$ in the order 

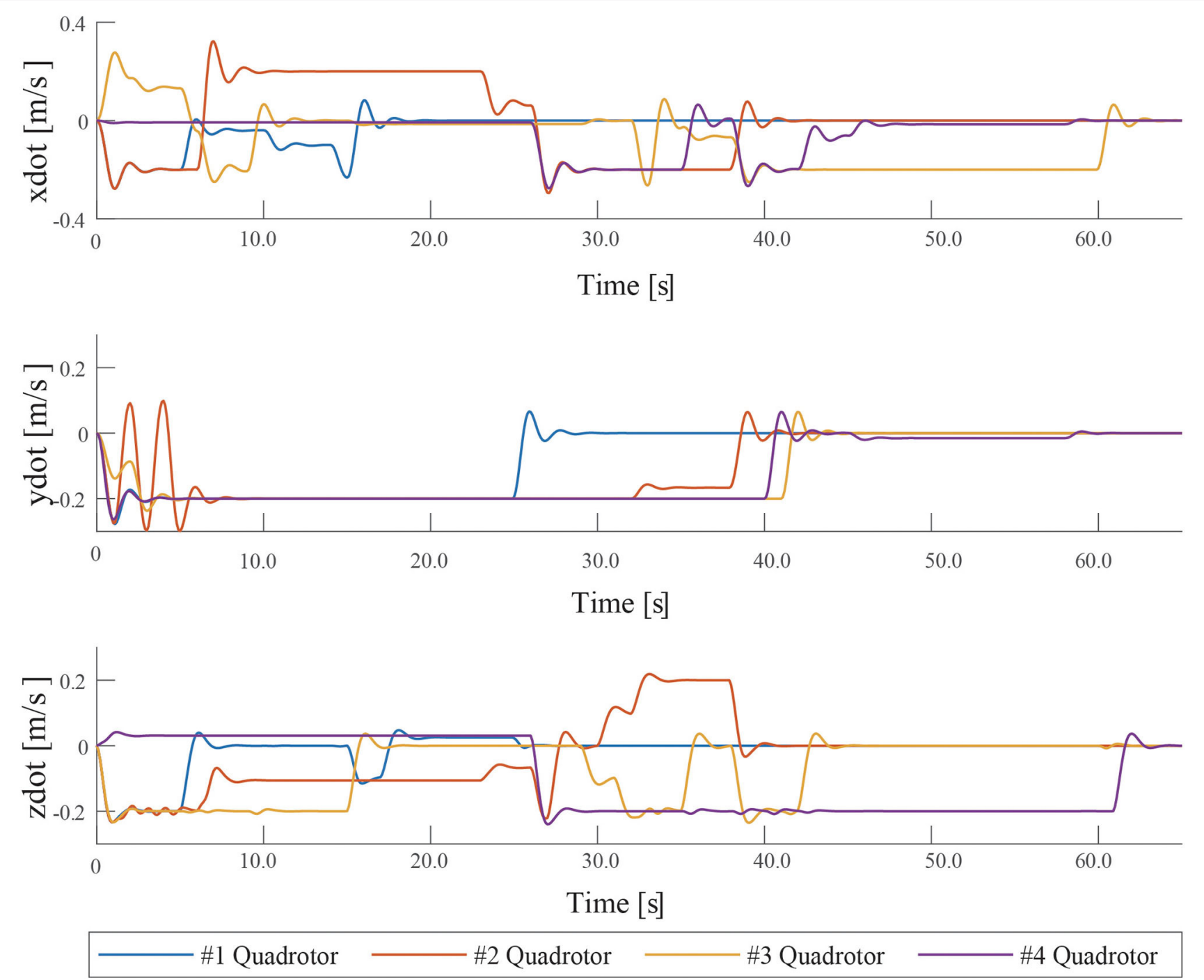

FIGURE 8 | The snapshot of path planning and tracking for UAVs in 3-D environments at $65 \mathrm{~s}$.

shown in Figure 10. In order to better observe the experimental results, only two UAVs are used for the experiment. However, the proposed MPPTM can carry out online path planning and tracking for more robot individuals in an environment with dynamic obstacles.

Figure 10 shows that two quadrotors can avoid dynamic obstacles to capture targets during the path planning and tracking. The experimental result indicates that MPPTM carries out online path planning and tracking of MRS in an environment with dynamic obstacles.

\section{DISCUSSION}

Several comparative experiments on the proposed path optimizer and the proposed path planner are given in this section.

\subsection{The Performance of the Proposed Path Optimizer}

By using two different path optimizers, the actual paths and the desired paths are recoded in the experiment in section 3.2. The tracking error is the sum of the errors between the actual path and the desired path on the $X, Y$, and $Z$ axes. The errors of the quadrotors are shown in Figure 11.

An indicator, defined as Equation (12), is given to measure the tracking error of UAVs during online path planning and tracking, where $R$ is the number of quadrotors in UAVs, $\operatorname{error}_{i}(t)$ is the error the $i_{\text {th }}$ quadrotor, and $t$ is the time.

$$
E=\frac{1}{R} \sum_{i=1}^{R} \int_{0}^{+\infty} \operatorname{error}_{i}(t) d t
$$

In order to eliminate the influence of a series of factors as far as possible, such as wind speed, battery status, and measurement 

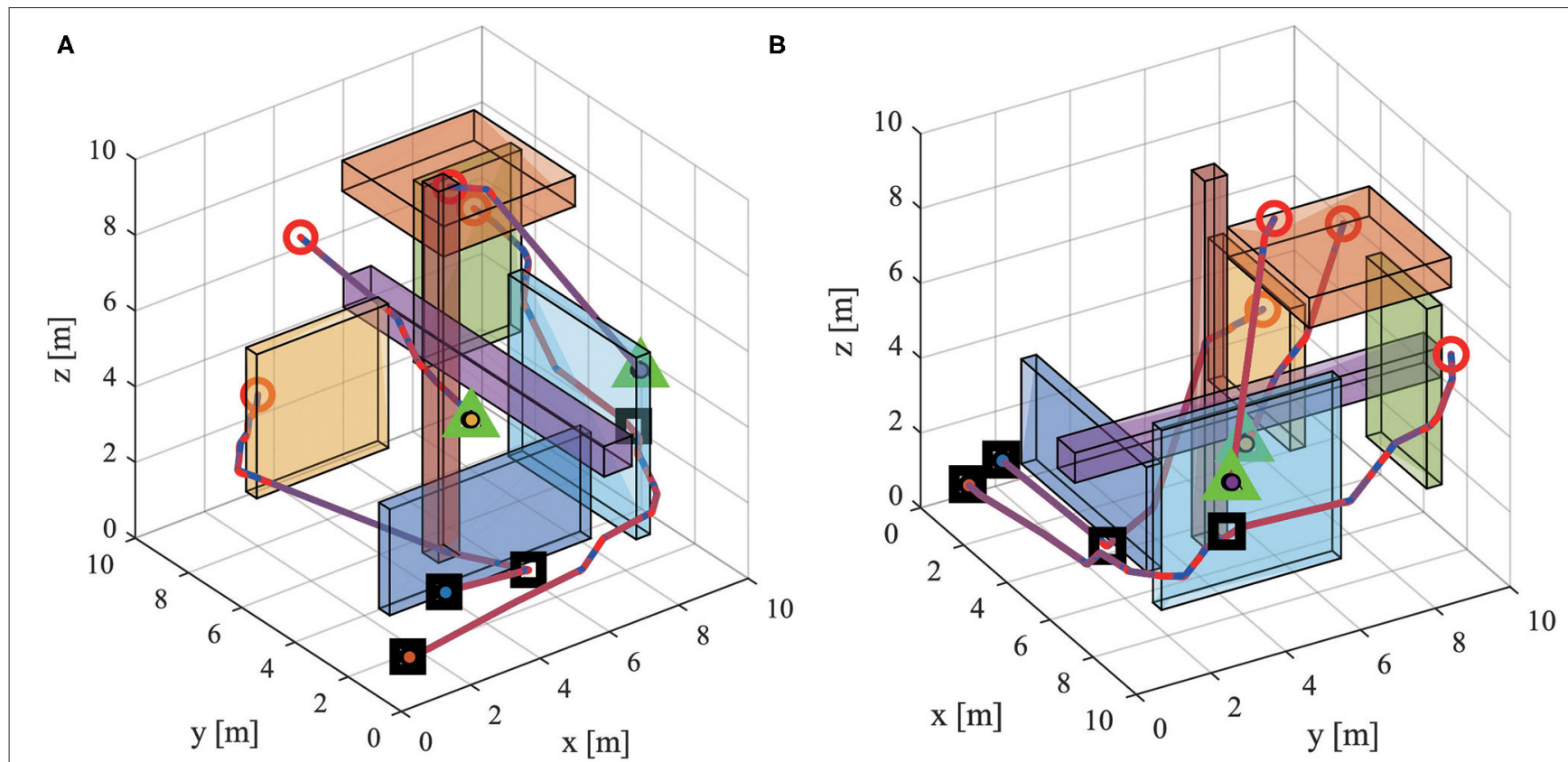

FIGURE 9 | The snapshot of path planning and tracking with quadrotor fault in 3-D environments. (A) Observation from view (-38, 30). (B) Observation from view (60, 30). (Green triangle represents the position of quadrotor fault. The meaning of other markers are the same as that in Figure 6).

A

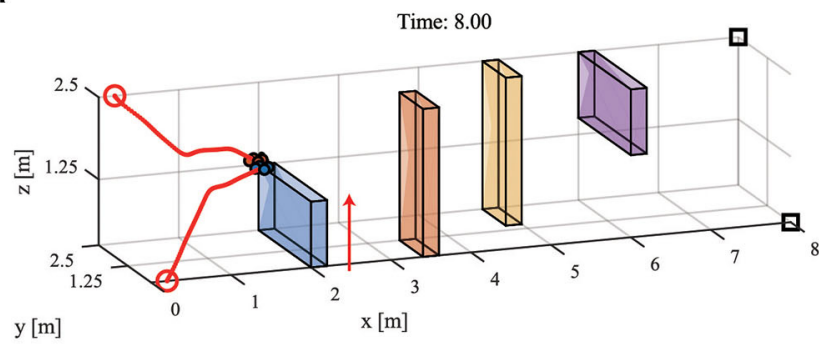

C

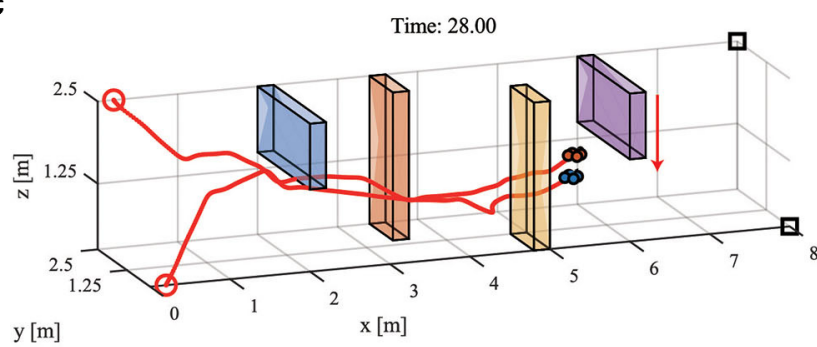

B

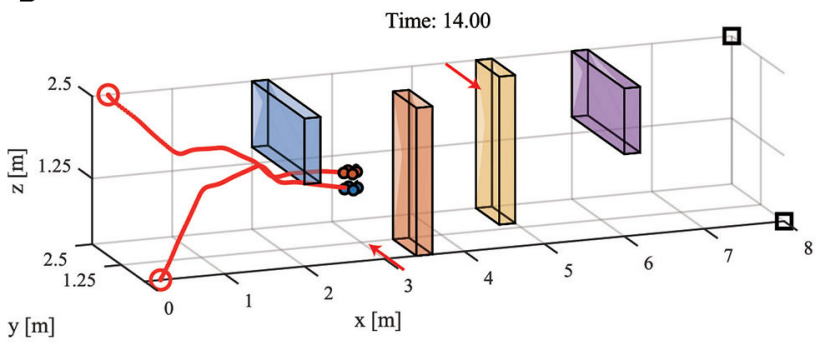

D

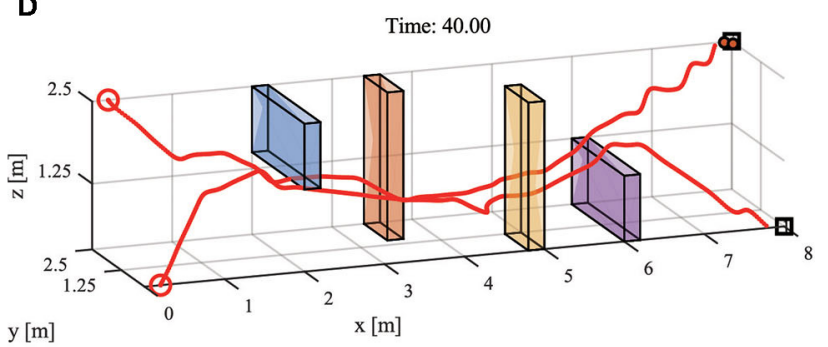

FIGURE 10 | The path planning and tracking with dynamic obstacles in 3-D environments. (A) The snapshot at 8 s. (B) The snapshot at $14 \mathrm{~s}$. (C) The snapshot at 28 s. (D) The snapshot at 40 s. (Red arrow represents the direction of obstacles movement. The meaning of other markers are the same as that in Figure 6).

error, we test 20 experiments and collect the data of tracking error. These data are shown in Table 1.

Table 1 shows that, compared with the original path optimizer, the proposed path optimizer can reduce the tracking error of UAVs during online path planning and tracking.

\subsection{The Time Performance of the Proposed Path Planner}

By using the proposed neural activity algorithm, the proposed path planner has better time performance than other approaches (Li et al., 2009; Yi and Zhu, 2013; Sun et al., 2019; 


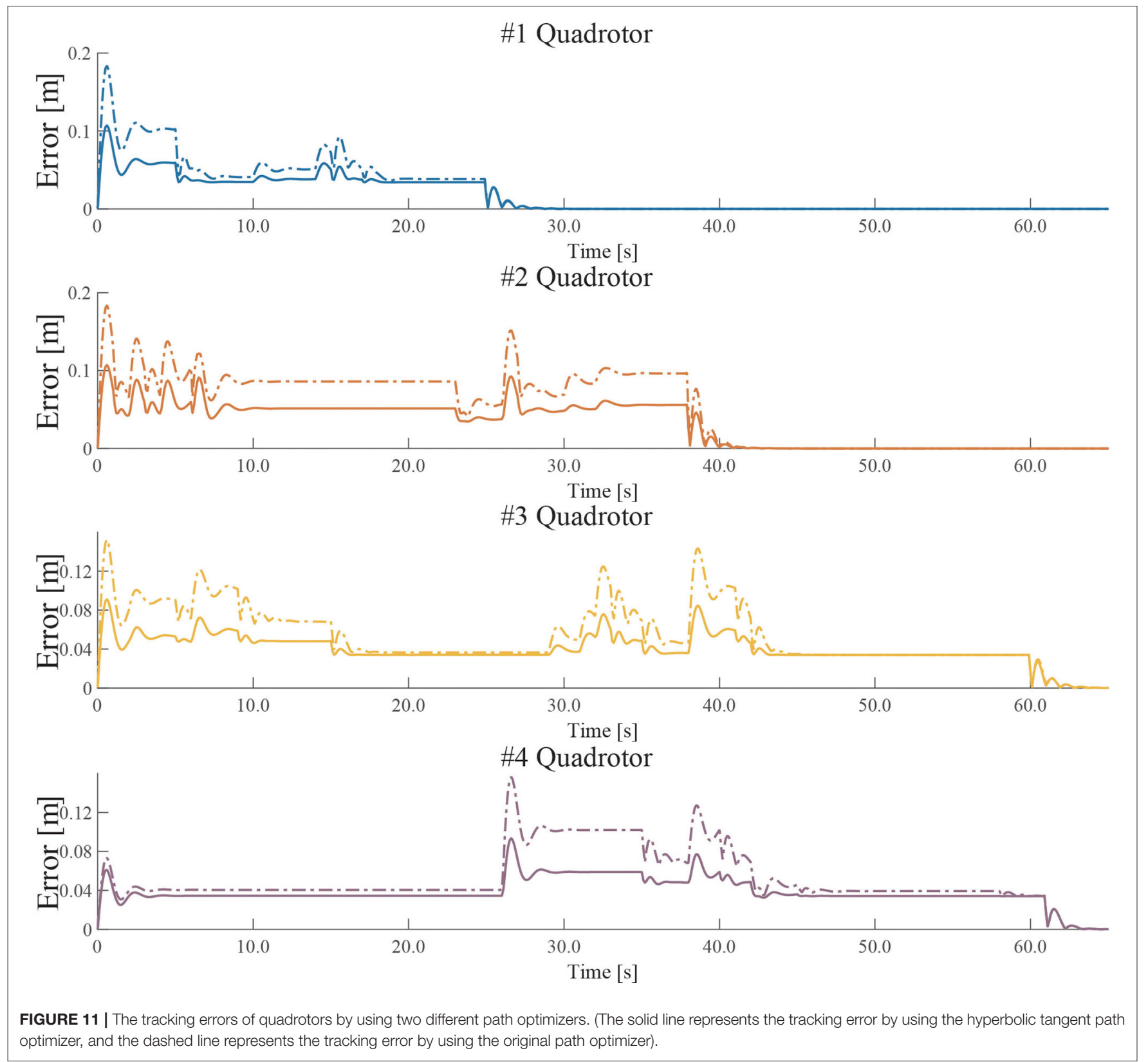

TABLE 1 | The comparison of tracking error of unmanned aerial vehicle (UAVS).

\begin{tabular}{lccc}
\hline Case & $\operatorname{Max}(\boldsymbol{E})$ & $\operatorname{Min}(\boldsymbol{E})$ & $\boldsymbol{A v g}(\boldsymbol{E})$ \\
\hline The original path optimizer & 1.5669 & 1.3768 & 1.4797 \\
The proposed path optimizer & $\mathbf{1 . 0 4 5 3}$ & $\mathbf{0 . 9 7 6 5}$ & $\mathbf{1 . 0 2 7 8}$
\end{tabular}

Bold indicates that this indicator is the most superior to other cases.

Ni et al., 2020; Zhu et al., 2021). The comparative simulations are coded by Matlab, which is run on the PC with Intel i7-7700, 28GB ROM, and Win10 OS. The average time consumptions of single path planning by using different approaches and different sizes are shown in Table 2.
Table 2 indicates that the time performance of the proposed path planner is better than other approaches, and it is insensitive to the number of robots in MRS. The proposed path planner has excellent time performance, which makes it very suitable for superscalar pipelining mode in the proposed path planner of MPPTM for online path planning and tracking in 3D environments.

\section{CONCLUSION}

The proposed model, MPPTM, can deal with task-level and control-level problems for path planning and tracking of MRS in 3-D environments. During the online path planning and tracking, our proposed model only needs to obtain the position 
TABLE 2 | The average time consumptions of different approaches (s).

\begin{tabular}{cccccc}
\hline \multicolumn{1}{c}{ Case } & Size & $\mathbf{R = 4}$ & $\mathbf{R = 8}$ & $\mathbf{R = 1 2}$ & $\mathbf{R = 1 6}$ \\
\hline \multirow{2}{*}{ Our approach } & $30 \times 30$ & $\mathbf{0 . 1 7 3 8}$ & $\mathbf{0 . 1 8 3 1}$ & $\mathbf{0 . 1 6 8 6}$ & $\mathbf{0 . 1 8 2 6}$ \\
& $50 \times 50$ & $\mathbf{0 . 8 5 6 3}$ & $\mathbf{0 . 8 3 2 2}$ & $\mathbf{0 . 8 6 4 7}$ & $\mathbf{0 . 8 5 3 4}$ \\
Li et al. (2009) & $30 \times 30$ & 3.5184 & 7.4610 & 9.8962 & 15.7595 \\
& $50 \times 50$ & 18.3248 & 37.6820 & 46.9013 & 74.6896 \\
Yi and Zhu (2013) & $30 \times 30$ & 0.9502 & 0.8306 & 0.9999 & 0.9882 \\
& $50 \times 50$ & 5.0008 & 3.9363 & 5.0498 & 4.9412 \\
Sun et al. (2019) & $30 \times 30$ & 2.8010 & 5.6704 & 6.8507 & 10.5158 \\
& $50 \times 50$ & 13.2122 & 25.7745 & 34.4256 & 52.0586 \\
Ni et al. (2020) & $30 \times 30$ & 2.563 & 2.7275 & 2.9789 & 3.4007 \\
& $50 \times 50$ & 12.0897 & 13.8657 & 15.0516 & 18.0413 \\
Zhu et al. (2021) & 30X30 & 2.2681 & 4.5916 & 5.5473 & 8.5152 \\
& $50 \times 50$ & 10.6986 & 20.871 & 27.8763 & 42.1546
\end{tabular}

Bold indicates that this indicator is the most superior to other cases.

of MRS instead of relying on the complex sensor data of individual robots to plan paths. The cost of MRS equipped with such complex sensors is huge. Therefore, our proposed model, MPPTM, is a low-cost solution for the online path planning of MRS. Based on the UWB positioning system, MPPTM can carry out online path planning and high-accuracy path tracking for MRS in indoor or outdoor environments. It is suitable for application in manufacturing plants and industrial parks.

\section{REFERENCES}

Ali, H., Gong, D., Wang, M., and Dai, X. (2020). Path planning of mobile robot with improved ant colony algorithm and mdp to produce smooth trajectory in grid-based environment. Front. Neurorobot. 14:44. doi: 10.3389/fnbot.2020.00044

Ali, S., Mehmood, F., Dancey, D., Ayaz, Y., Khan, M. J., Naseer, N., et al. (2019). An adaptive multi-robot therapy for improving joint attention and imitation of asd children. IEEE Access 7, 81808-81825. doi: 10.1109/ACCESS.2019.29 23678

Chen, M., and Zhu, D. (2019). A workload balanced algorithm for task assignment and path planning of inhomogeneous autonomous underwater vehicle system. IEEE Trans. Cogn. Develop. Syst. 11, 483-493. doi: 10.1109/TCDS.2018.2866984

Chen, Z., Alonso-Mora, J., Bai, X., Harabor, D. D., and Stuckey, P. J. (2021). Integrated task assignment and path planning for capacitated multi-agent pickup and delivery. IEEE Robot. Autom. Lett. 6, 5816-5823. doi: 10.1109/LRA.2021.3074883

Dai, X., Long, S., Zhang, Z., and Gong, D. (2019). Mobile robot path planning based on ant colony algorithm with $\mathrm{a}^{*}$ heuristic method. Front. Neurorobot. 13:15. doi: 10.3389/fnbot.2019.00015

Dong, R., Liu, C., Wang, X., and Han, X. (2020). 3d path planning of uavs for transmission lines inspection. Int. J. Robot. Autom. 35, 146-158. doi: 10.2316/j.2020.206-0146

Han, S. D., and Yu, J. (2020). Ddm: Fast near-optimal multi-robot path planning using diversified-path and optimal sub-problem solution database heuristics. IEEE Robot. Autom. Lett. 5, 1350-1357. doi: 10.1109/LRA.2020.2967326

Koutras, D. I., Kapoutsis, A. C., and Kosmatopoulos, E. B. (2020). Autonomous and cooperative design of the monitor positions for a team of uavs to maximize the quantity and quality of detected objects. IEEE Robot. Autom. Lett. 5, 4986-4993. doi: 10.1109/LRA.2020.3004780

Krizmancic, M., Arbanas, B., Petrovic, T., Petric, F., and Bogdan, S. (2020). Cooperative aerial-ground multi-robot system for automated construction
Real experiments in this article demonstrate the applicability and effectiveness of the proposed model. In this model, the proposed path planner has excellent time performance to meet the requirement of superscalar pipelining mode. Meanwhile, the proposed path optimizer can guarantee the high-accuracy tracking of UAVs.

\section{DATA AVAILABILITY STATEMENT}

The original contributions presented in the study are included in the article/supplementary material, further inquiries can be directed to the corresponding author/s.

\section{AUTHOR CONTRIBUTIONS}

$\mathrm{XY}$ and $\mathrm{AZ}$ contributed to the conception of the study. $\mathrm{XY}$ performed the experiment, the data analyses and wrote the manuscript. AZ contributed significantly to analysis and manuscript preparation. SY helped perform the analysis with constructive discussions. All authors listed have made a direct and intellectual contribution to the study, and approved it for publication.

\section{FUNDING}

This study was supported by National Natural Science Foundation of China (61273354) and Shenzhen stability support program for university (20200812112522002). tasks. IEEE Robot. Autom. Lett. 5, 798-805. doi: 10.1109/LRA.2020. 2965855

Li, H., Yang, S. X., and Seto, M. L. (2009). Neural-network-based path planning for a multirobot system with moving obstacles. IEEE Trans. Syst. Man Cybern. Part C (Appl. Rev.) 39, 410-419. doi: 10.1109/TSMCC.2009.2 020789

Ma, L. (2020). Cooperative target tracking in balanced circular formation with time-varying radius. Int. J. Robot. Autom. 35, 86-100. doi: 10.2316/J.2020.206-0086

Mehmood, F., Ayaz, Y., Ali, S., De Cassia Amadeu, R., and Sadia, H. (2019). Dominance in visual space of asd children using multi-robot joint attention integrated distributed imitation system. IEEE Access 7, 168815-168827. doi: 10.1109/ACCESS.2019.2951366

Morita, T., Kashiwagi, N., Yorozu, A., Walch, M., Suzuki, H., Karagiannis, D., et al. (2018). "Practice of multi-robot teahouse based on printeps and evaluation of service quality," in 2018 IEEE 42nd Annual Computer Software and Applications Conference (COMPSAC), Vol. 02, (Tokyo) 147-152.

Ni, J., Wang, X., Tang, M., Cao, W., Shi, P., and Yang, S. X. (2020). An improved real-time path planning method based on dragonfly algorithm for heterogeneous multi-robot system. IEEE Access 8, 140558-140568. doi: 10.1109/ACCESS.2020.3012886

Park, S., Min, Y., Ha, J., Cho, D., and Choi, H. (2019). A distributed admm approach to non-myopic path planning for multi-target tracking. IEEE Access 7, 163589-163603. doi: 10.1109/ACCESS.2019.2952235

Penin, B., Giordano, P. R., and Chaumette, F. (2018). Vision-based reactive planning for aggressive target tracking while avoiding collisions and occlusions. IEEE Robot. Autom. Lett. 3, 3725-3732. doi: 10.1109/LRA.2018. 2856526

Queralta, J. P., Taipalmaa, J., Can Pullinen, B., Sarker, V. K., Nguyen Gia, T., Tenhunen, H., et al. (2020). Collaborative multi-robot search and rescue: Planning, coordination, perception, and active vision. IEEE Access 8, 191617191643. doi: 10.1109/ACCESS.2020.3030190 
Rubí, B., Pérez, R., and Morcego, B. (2019). A survey of path following control strategies for uavs focused on quadrotors. J. Intell. Robot. Syst. 1-25. doi: 10.1007/s10846-019-01085-z

Silic, M. and Mohseni, K. (2019). Field deployment of a plume monitoring uav flock. IEEE Robot. Autom. Lett. 4, 769-775. doi: 10.1109/LRA.2019.2893420

Sun, B., Zhu, D., Tian, C., and Luo, C. (2019). Complete coverage autonomous underwater vehicles path planning based on glasius bio-inspired neural network algorithm for discrete and centralized programming. IEEE Trans. Cogn. Develop. Syst. 11, 73-84. doi: 10.1109/TCDS.2018.2810235

Wang, J., Chi, W., Li, C., Wang, C., and Meng, M. Q. H. (2020a). Neural $\mathrm{rrt}^{*}$ : Learning-based optimal path planning. IEEE Trans. Autom. Sci. Eng. 17, 1748-1758. doi: 10.1109/TASE.2020.2976560

Wang, J., Wang, J., and Che, H. (2020b). Task assignment for multivehicle systems based on collaborative neurodynamic optimization. IEEE Trans. Neural Netw. Learn. Syst. 31, 1145-1154. doi: 10.1109/TNNLS.2019.2918984

$\mathrm{Xu}, \mathrm{T}$., and Tang, L. (2021). Adoption of machine learning algorithm-based intelligent basketball training robot in athlete injury prevention. Front. Neurorobot. 14:117. doi: 10.3389/fnbot.2020.620378

Yi, X., and Zhu, A. (2013). "An improved neuro-dynamics-based approach to online path planning for multi-robots in unknown dynamic environments," in 2013 IEEE International Conference on Robotics and Biomimetics (ROBIO), 1-6.

Yi, X., Zhu, A., Yang, S. X., and Luo, C. (2017). A bio-inspired approach to task assignment of swarm robots in 3-d dynamic environments. IEEE Trans. Cybern. 47, 974-983. doi: 10.1109/TCYB.2016.2535153

Yordanova, V., and Gips, B. (2020). Coverage path planning with track spacing adaptation for autonomous underwater vehicles. IEEE Robot. Autom. Lett. 5, 4774-4780. doi: 10.1109/LRA.2020.3003886

Yu, J., Dong, X., Li, Q., and Ren, Z. (2018). Practical time-varying formation tracking for second-order nonlinear multiagent systems with multiple leaders using adaptive neural networks. IEEE Trans. Neural Netw. Learn. Syst. 29, 6015-6025. doi: 10.1109/TNNLS.2018. 2817880
Yu, J., Su, Y., and Liao, Y. (2020). The path planning of mobile robot by neural networks and hierarchical reinforcement learning. Front. Neurorobot. 14:63. doi: 10.3389/fnbot.20 20.00063

Zeng, Z., Lian, L., Sammut, K., He, F., Tang, Y., and Lammas, A. (2015). A survey on path planning for persistent autonomy of autonomous underwater vehicles. Ocean Eng. 110, 303-313. doi: 10.1016/j.oceaneng.2015.10.007

Zhou, L., Tzoumas, V., Pappas, G. J., and Tokekar, P. (2018). Resilient active target tracking with multiple robots. IEEE Robot. Autom. Lett. 4, 129-136. doi: 10.1109/LRA.2018.2881296

Zhu, D., Zhou, B., and Yang, S. X. (2021). A novel algorithm of multi-auvs task assignment and path planning based on biologically inspired neural network map. IEEE Trans. Intell. Veh. 6, 333-342. doi: 10.1109/TIV.2020.30 29369

Conflict of Interest: The authors declare that the research was conducted in the absence of any commercial or financial relationships that could be construed as a potential conflict of interest.

Publisher's Note: All claims expressed in this article are solely those of the authors and do not necessarily represent those of their affiliated organizations, or those of the publisher, the editors and the reviewers. Any product that may be evaluated in this article, or claim that may be made by its manufacturer, is not guaranteed or endorsed by the publisher.

Copyright (c) 2022 Yi, Zhu and Yang. This is an open-access article distributed under the terms of the Creative Commons Attribution License (CC BY). The use, distribution or reproduction in other forums is permitted, provided the original author(s) and the copyright owner(s) are credited and that the original publication in this journal is cited, in accordance with accepted academic practice. No use, distribution or reproduction is permitted which does not comply with these terms. 